\title{
Impact of patatin-like phospholipase domain-containing protein 3 (rs738409-G) polymorphism on post-transplant outcomes after liver transplantation for alcohol-related liver disease
}

\author{
Tae YOO', Kwang Woong LEE*2, Nam-Joon $\mathrm{YI}^{2}$, Suk Kyun HONG ${ }^{2}$, Kyung-Suk SUH ${ }^{2}$ \\ 'Department of Surgery, Dongtan Sacred Heart Hospital, Hwaseong, Korea \\ ${ }^{2}$ Department of Surgery, Seoul National University College of Medicine, Seoul, Korea
}

Introduction: We aimed to evaluate the association between patatin-like phospholipase domain-containing protein 3 (PNPLA3) polymorphism and post-liver transplantation (LT) outcomes related to alcohol relapse (AR).

Methods: We retrospectively analyzed data from patients receiving LT for alcoholic liver disease (ALD) from 04/2014 to 12/2017. Liver-related clinical outcomes were assessed by the gamma-glutamyltransferase (GGT) level and alcohol-related liver failure (ARLF). Genotyping was performed using prospectively collected DNA samples in both donors and recipients.

Results: A total of 83 recipients were enrolled. Post-LT AR occurred in 31 patients (37.3\%). Thirty-one patients (14 AR, 9 abstainers) showed elevated GGT levels, and 3 AR patients experienced ARLF. In the multivariate analysis, rs738409-G allele carrier and heavy drinking (HRAR score $\geq 4$ ) were independent risk factors for elevated GGT levels (odds ratio $[\mathrm{OR}]=8.69, \mathrm{p}<0.01 ; \mathrm{OR}=13.07, p=0.01$ ) and ARLF ( $\mathrm{OR}=4.52, p=0.04 ; \mathrm{OR}=19.62, p=0.03)$. Among 15 heavy AR patients, being an rs738409-G allele carrier was related to GGT elevation $(p=0.03)$ and ARLF $(p=0.04)$, but it was not to GGT elevation in mild drinkers $(\mathrm{n}=16)$ or abstainers $(\mathrm{n}=52)$.

Conclusions: PNPLA3 polymorphism of the recipient genotype can independently affect the post-LT prognosis of LT patients for ALD, especially in heavy AR patients. Therefore, strong abstinence education is recommended in patients with this single nucleotide polymorphism. 